\title{
Simultaneous two-wavelength transmission quantitative phase microscopy with a color camera
}

\author{
Matthew T. Rinehart, ${ }^{1, *}$ Natan T. Shaked, ${ }^{1}$ Nathan J. Jenness, ${ }^{2}$ Robert L. Clark, ${ }^{2}$ and Adam Wax ${ }^{1}$ \\ ${ }^{1}$ Department of Biomedical Engineering, Fitzpatrick Institute for Photonics, Duke University, Durham, North Carolina 27708, USA \\ ${ }^{2}$ Department of Mechanical Engineering and Materials Science, Duke University, Durham, North Carolina 27708, USA \\ *Corresponding author: matt.rinehart@duke.edu
}

Received April 13, 2010; revised June 11, 2010; accepted June 24, 2010; posted July 8, 2010 (Doc. ID 126768); published July 28, 2010

\begin{abstract}
We present a quantitative phase microscopy method that uses a Bayer mosaic color camera to simultaneously acquire off-axis interferograms in transmission mode at two distinct wavelengths. Wrapped phase information is processed using a two-wavelength algorithm to extend the range of the optical path delay measurements that can be detected using a single temporal acquisition. We experimentally demonstrate this technique by acquiring the phase profiles of optically clear microstructures without $2 \pi$ ambiguities. In addition, the phase noise contribution arising from spectral channel crosstalk on the color camera is quantified. @ 2010 Optical Society of America OCIS codes: $090.5694,090.4220,100.5088,180.3170$.
\end{abstract}

Transmission-geometry quantitative phase microscopy (QPM) has been developed for three-dimensional measurement and characterization of a wide variety of transparent samples, such as transparent optical elements (e.g., microlens arrays) [1], optical fibers [2], and living cells $[3,4]$. This interferometric measurement of optical path delays (OPDs) provides quantitative contrast arising from both the physical height of the sample and its refractive index changes. While QPM provides diffractionlimited lateral resolution and nanometer-scale axial resolution of OPDs, the axial range over which the phase can be unambiguously determined is limited to $2 \pi$, which corresponds to one full wavelength of the illumination light.

To solve the $2 \pi$ ambiguities in the acquired phase profiles and determine the correct relative OPDs across a field of view, two-dimensional unwrapping algorithms are typically employed. Classic unwrapping algorithms are based on gradient minimization, which adds multiples of $2 \pi$ at specific points in the phase map [5] . While these algorithms can accurately recover a smooth and slowly varying phase map, they frequently fail to accurately reconstruct objects containing a phase difference greater than $\pi$ between adjacent image pixels.

An alternative approach to overcoming the limitations of phase unwrapping is the use of multiple illumination wavelengths. By processing the phase profiles obtained from different wavelengths, it becomes possible to synthesize a single phase profile that is unambiguous over the range of the "beat" wavelength [6,7], which is larger than the unambiguous range that results from using a single wavelength independently. Because this approach is based on an analytical solution and not an iterative numerical method, two-wavelength phase unwrapping is particularly useful when classic unwrapping algorithms fail to correctly unwrap across sharp phase discontinuities.

Two-wavelength phase unwrapping has been previously employed in reflection-geometry QPM using both sequential $[8]$ and simultaneous $[9,10]$ illumination/ detection schemes to accurately reconstruct surface profiles of highly reflective structures. Two-wavelength phase unwrapping has also been used for transmission- geometry phase microscopy $[1,11]$; however, these methods use sequential illumination and detection, which require multiple temporal acquisitions and careful synchronization of illumination switching and image acquisition. In this Letter, we present a transmissiongeometry optical microscope that uses a Bayer mosaic color CCD camera to capture off-axis interferograms at each of two wavelengths in a single exposure. This method simplifies both the optical setup and the process of data acquisition substantially, and guarantees that the unwrapping process will not be affected by differing motion artifacts. The wrapped phase information retrieved from these interferograms is input into a two-wavelength unwrapping algorithm to unambiguously reconstruct the OPD map.

Figure 1 presents the optical system, an off-axis interferometric transmission microscope based upon the Mach-Zehnder geometry. Light from two distinct wavelengths from separate laser sources, chosen to match the peak spectral responses of the Bayer pattern red and green channels, is coupled into a beam splitter (BS1) and aligned to follow identical optical paths. The two arms of the interferometer contain matched microscope objectives (MOs) that are aligned in $4 f$ configuration with lens L1 to magnify the sample while maintaining matched optical wavefronts. A tilt in the path of the reference arm produces a high frequency linear interference pattern in the image plane. A Bayer mosaic color camera (Fig. 1, inset) records an off-axis interferogram at each

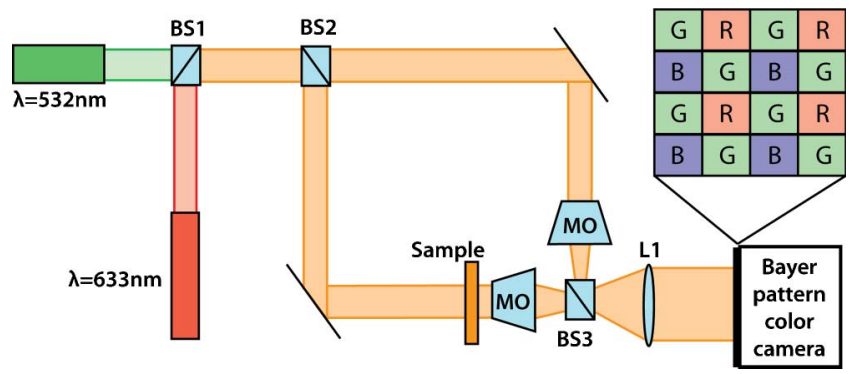

Fig. 1. (Color online) Optical system based on a modified Mach-Zehnder interferometric microscope with two illumination lasers. Inset, pixel filter layout of a Bayer mosaic color camera. 
wavelength simultaneously. Based on the Bayer mosaic pixel geometry, the red and green channels have larger spatial sampling periods than an equivalent grayscale detector; therefore, the system magnification necessary to avoid aliasing is higher for the color camera and results in a smaller field of view [12].

Quantitative OPD maps of microscopic samples are obtained by the following methods: First, off-axis interferograms are simultaneously acquired at both wavelengths and are separately detected by color channel. Digital spatial filtering in the Fourier domain is used to remove the contributions from the complex conjugate and autocorrelation terms, and the remaining frequency content is recentered in spatial frequency space to demodulate the signal $[13,14]$. Next, wrapped phase information at each wavelength is recovered by taking the angle argument of the complex data on a pixel-by-pixel basis. Finally, the OPD map at the synthetic beat wavelength is calculated and used as a guide to add the correct multiple of $2 \pi$ to each point of the wrapped OPD map for one of the individual wavelengths [8] chosen to be the shorter wavelength, since it offers slightly better diffraction-limited resolution. The resulting OPD map is unambiguous over the range of the synthetic beat wavelength and retains the low noise level of the original single wavelength wrapped image.

In our experiments, a red $\mathrm{He}-\mathrm{Ne}$ laser $\left(\lambda_{1}=\right.$ $632.8 \mathrm{~nm}$ ) and a green diode-pumped solid state (DPSS) laser $\left(\lambda_{2}=532 \mathrm{~nm}\right)$ were used as illumination sources, which together produce a synthetic beat wavelength, $\Lambda$, of $3.334 \mu \mathrm{m}\left(\Lambda=\lambda_{1} \lambda_{2} /\left|\lambda_{1}-\lambda_{2}\right|\right)$ [8]. Imaging was accomplished using $40 \times$ objectives with an NA of 0.66 in $4 f$ configuration with a $150 \mathrm{~mm}$ focal length lens, providing a sample magnification of $33.3 \times$ at the image plane. The interferometric signal was recorded using a Bayer mosaic color camera (12 bit CoolSNAP $c f$, Photometrics) with $1392 \times 10404.65 \mu \mathrm{m}$ square pixels. The diffractionlimited resolution of the system is $0.492 \mu \mathrm{m}$ and $0.585 \mu \mathrm{m}$ $(d=0.61 \lambda / \mathrm{NA})$ for the green and red illuminations, respectively.

Based on the spectral response curves of the camera, there is a $5 \%$ predicted intensity crosstalk between the red and green channels, which could slightly increase the phase noise in each interferogram when both are captured simultaneously. When uniformly illuminated with only the red laser, the red-to-green crosstalk was found to be $4.3 \%$; when illuminating with only the green laser, the green-to-red crosstalk was found to be $5.4 \%$. These crosstalk figures indicate that a Bayer mosaic filter provides high wavelength selectivity. To assess the effects of intensity crosstalk on phase measurement, interferograms with no sample were acquired individually with either red or green illumination, and then simultaneously with both illumination sources. OPD maps were calculated using the methods described above, and the OPD standard deviation, $\sigma$, of a $14 \times 14 \mu \mathrm{m}$ region was compared. Comparing individual to simultaneous illumination, $\sigma_{\text {red }}$ changed from 15.5 to $15.9 \mathrm{~nm}$, and $\sigma_{\text {green }}$ changed from 13.7 to $13.8 \mathrm{~nm}$. Since neither $\sigma_{\text {red }}$ nor $\sigma_{\text {green }}$ increased significantly, we conclude that the Bayer mosaic pattern is highly effective for separating simultaneously acquired interferograms at two wavelengths.
To verify axial sensitivity obtained with this method, we measured the phase profile of a $20 \mu \mathrm{m}$ diameter polystyrene microsphere (Duke Scientific) immersed in index-matching oil $(n=1.515)$. The simulated twodimensional OPDs induced by an equal-diameter ideal sphere were subtracted from the OPD measurements, yielding an rms deviation of $17.312 \mathrm{~nm}$, or $\sim \lambda / 30$, across the entire area covered by the microsphere. This error incorporates not only error arising from phase noise but also any surface imperfections of the microsphere, and therefore represents an upper bound on the axial sensitivity of this system. The diameter as calculated from the measured OPD at the center of the bead was found to be $20.105 \mu \mathrm{m}$, which is in close agreement with the National Institute of Standards and Technology (NIST) -certified diameter of this microsphere sample of $19.99 \pm 0.20 \mu \mathrm{m}$.

Additional experimental validation of simultaneous two-wavelength transmission QPM with a Bayer mosaic CCD was performed by measuring phase profiles of optically clear microstructures. These microstructures were created by maskless holographic micropatterning of UV-cured optical adhesive (NOA63, Norland Products) onto glass microscope coverslips [15]. We first imaged the microstructures with a scanning electron microscope (SEM) in both en face Fig. 2(a) and $45^{\circ}$ tilt Fig. 2(b) geometries in order to visualize the structures. Note that while these SEM images allow visualization of the structures, quantitative axial measurements can only be inferred. Next, these microstructures $(n=1.56)$ were immersed in index-matching oil $(n=1.515)$, producing a relative refractive index difference of $\Delta n=0.045$ and imaged using the QPM methods described above. A background phase image was acquired from an adjacent area containing only the coverslip and index-matching oil and used to correct for wavefront curvature in the imaging system before applying phase unwrapping techniques.

To demonstrate the efficacy of our technique, we compared the results of a well-known quality-map guided phase unwrapping algorithm [6] to two-wavelength unwrapping. The quality-map guided algorithm iteratively adds multiples of $2 \pi$ to the wrapped phase until the global phase gradient has been minimized. Figure 3(a) shows the OPD map produced using this method, and Fig. 3(c) shows a cross-section plot of the calculated physical object height. One of the four objects appears to be accurately reconstructed at a larger OPD than the background, while the other three objects are almost indistinguishable from the background. When examining the
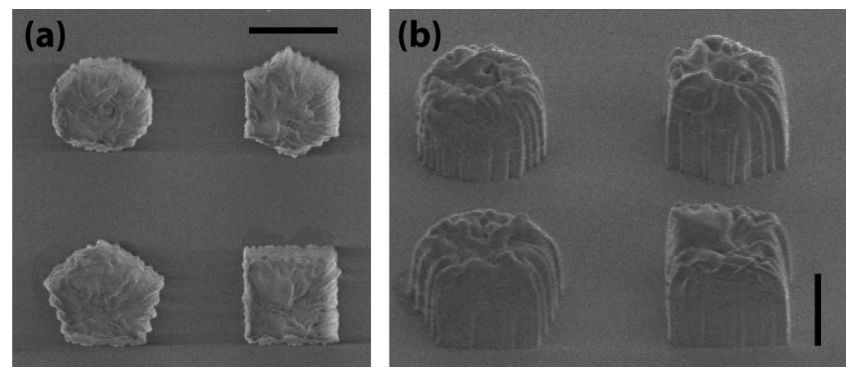

Fig. 2. SEM images of optical adhesive microstructures (2170 $\times$ magnification): (a) en face view with $15 \mu \mathrm{m}$ lateral scale bar; (b) $45^{\circ}$ tilt view with $15 \mu \mathrm{m}$ vertical scale bar. 


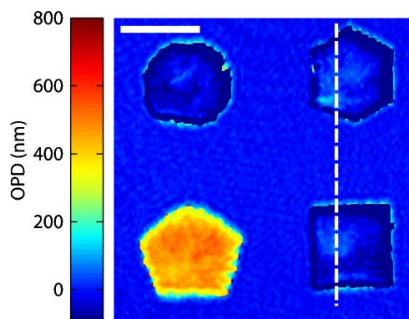

(a)

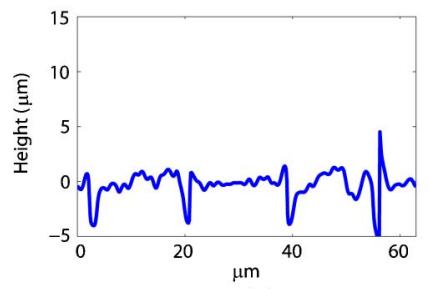

(c)

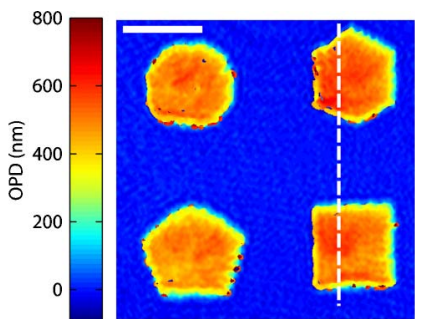

(b)

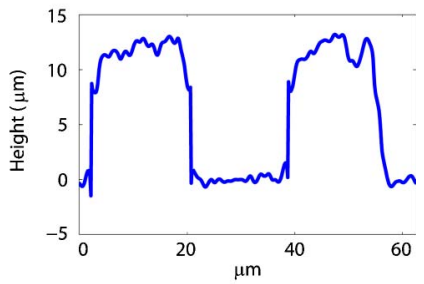

(d)
Fig. 3. (Color online) Microstructure OPD maps and profiles: (a) $532 \mathrm{~nm}$ OPD map after quality-map guided unwrapping, $15 \mu \mathrm{m}$ lateral scale bar; (b) $532 \mathrm{~nm}$ OPD map after twowavelength unwrapping, $15 \mu \mathrm{m}$ lateral scale bar; (c) incorrect object height profile, from the dotted line in (a); (d) object height profile from two-wavelength unwrapping, from the dotted line in (b). The quantitative height measurements obtained from QPM agree with the SEM images in Fig. 2.

SEM images from Fig. 2, it is clear that the quality-map guided unwrapping of the latter structures is incorrect. The difference in unwrapping algorithm success among the objects is due to variability in the edge slope and true height of the fabricated microstructures; erroneous object unwrapping likely arises from microstructures with a significant number of sharp edges, where the OPD changes by more than $\pi$ between neighboring pixels.

Figure 3(b) shows the results when using our simultaneous two-wavelength unwrapping approach in which the beat wavelength map has been used as a guide for adding multiples of $2 \pi$ to the original green illumination wrapped phase map. A specific cross-section plot of the physical object height (after dividing by $\Delta n$ ) from twowavelength unwrapping is shown in Fig. 3(d). As can be seen from Fig. 3(d), the object heights are between 10 and $15 \mu \mathrm{m}$, which is in agreement with estimates from the SEM images in Fig. 2. The remaining errors at the edges of the microstructures arise from high local noise, which causes an extra multiple of $2 \pi$ to be added to the single wavelength phase map in the final refinement process $[9,11]$.

In summary, we have presented a method of using the channels of a Bayer mosaic color camera for simultaneously capturing interferograms at multiple distinct illumination wavelengths. Experimental results confirm that color channel crosstalk is minimal and does not noticeably affect two-wavelength unwrapping of recovered phase information. Because this technique extends the measurement range of transmission-geometry QPM using only a single acquisition, it should be especially useful for analysis of transparent samples in which dynamic processes are of interest, such as microscopic biological phenomena in microfluidic devices or microchip assays. Although sequential speed of acquisition with a monochromatic camera and gating of the illumination source can compete with the speed of acquisition of a single exposure from a color camera seen here, our technique is more useful for imaging highly dynamic samples, as will be explored in our future work.

The authors acknowledge the support of the McChesney Fellowship in Biomedical Engineering from Duke University, the Bikura Postdoctoral Fellowship from Israel, and National Science Foundation (NSF) grants CMMI-0609265 and CBET-0651622.

\section{References}

1. J. Müller, V. Kebbel, and W. Jüptner, Opt. Lasers Eng. 43 , 739 (2005).

2. A. Roberts, E. Ampem-Lassen, A. Barty, K. Nugent, G. Baxter, N. Dragomir, and S. Huntington, Opt. Lett. 27, 2061 (2002).

3. N. T. Shaked, J. D. Finan, F. Guilak, and A. Wax, J. Biomed. Opt. 15, 010505 (2010).

4. K. J. Chalut, W. J. Brown, and A. Wax, Opt. Express 15, 3047 (2007).

5. D. C. Ghiglia and M. D. Pritt, Two-Dimensional Phase Unwrapping: Theory, Algorithms, And Software (Wiley, 1998).

6. A. T. Forrester, W. E. Parkins, and E. Gerjuoy, Phys. Rev. 72, 728 (1947)

7. Y. Y. Cheng and J. C. Wyant, Appl. Opt. 23, 4539 (1984).

8. J. Gass, A. Dakoff, and M. Kim, Opt. Lett. 28, 1141 (2003).

9. J. Kühn, T. Colomb, F. Montfort, F. Charrière, Y. Emery, E. Cuche, P. Marquet, and C. Depeursinge, Opt. Express 15, 7231 (2007).

10. P. Picart, D. Mounier, and J. M. Desse, Opt. Lett. 33, 276 (2008).

11. P. Ferraro, L. Miccio, S. Grilli, M. Paturzo, S. De Nicola, A. Finizio, R. Osellame, and P. Laporta, Opt. Express 15, 14591 (2007).

12. D. J. Brady, Optical Imaging and Spectroscopy (Wiley, 2009), pp. 268-272.

13. E. Cuche, P. Marquet, and C. Depeursinge, Appl. Opt. 39 4070 (2000).

14. N. T. Shaked, Y. Zhu, M. T. Rinehart, and A. Wax, Opt. Express 17, 15585 (2009).

15. N. J. Jenness, R. T. Hill, A. Hucknall, A. Chilkoti, and R. L. Clark, Opt. Express 18, 11754 (2010). 